$\xi$

\title{
Series method to solve conformable fractional riccati differential equations
}

\author{
Mohammed Al Masalmeh * \\ ${ }^{1}$ Department of Math for foundation studies, Higher Colleges of Technology, UAE \\ *Corresponding author E-mail: Malmasalmeh@hct.ac.ae
}

\begin{abstract}
This paper investigates and states some properties of conformable fractional derivative, Further Study and applies the series solution for a case of conformable fractional Riccati deferential equation with variable coefficients "which is arising in stochastic games" or "hyperbolic boundary control." Recently, Prof. Roshdi Khalil introduced a new and interesting definition for the C F D, which is simpler than the previous definition in Caputo and Riemann-Liouville. It leads to many extensions of the classical theorems in calculus.
\end{abstract}

Keywords: Conformable Fractional Derivative; Conformable Fractional Integral; Power Series; Riccati Equation; Series Solution.

\section{Introduction and preliminaries}

Fractional calculus is a branch of mathematics that comes from the usual definition of calculus integral and derivative operators in much the same way as fractional exponents are an outgrowth of exponents with integer value.

In 1695 L'Hopital asked the question as to the meaning of $d^{n} y / d x^{n}$ if $\mathrm{n}=\frac{1}{2}$ that is "what if $\mathrm{n}$ is fractional?". Leibniz replied that " $\mathrm{d}^{\frac{1}{2}} \mathrm{x}$ will be equal to $\mathrm{x} \sqrt{\mathrm{dx}: \mathrm{x}}$ ". [1]

In the past few years' fractional calculus appeared as an important tool to deal with anomalous diffusion processes, which can be visualized as an ant in a labyrinth where the average square of the distance covered by the ant is $\left(\mathrm{x}^{2}(\mathrm{t})\right) \alpha \mathrm{t}^{2 \mu}$ where $\mu$ is a phenomenological constant for $\mu=\frac{1}{2}$ we have the ordinary diffusion processes. A more physical approach of anomalous diffusion processes has several applications in many field such as diffusion in porous media or long range correlation of DNA sequence.

On the other hand many numerical methods solutions of multi order fractional differential equations have been investigated for unique solutions and expectance of a solutions. For example, they have been used successfully to model frequency dependent damping behavior of many viscoelastic materials. Bagley and Torvik provided a review of work done in the area prior to 1980 [6], and showed that half-order fractional differential models describe the frequency dependence of damping materials very well. Other authors have demonstrated applications of fractional derivatives in the area of electrochemical processes, dielectric polarization, colored noise, viscoelastic materials, and chaos. [7]

Other scientist such as Mainardi, Rossikhin and Shitikiva apply the applications of fractional differential equations in general to solid mechanics, and modeling of viscoelastic damping. In addition Magin has produced produce a three part critical review of application of fractional calculus in bioengineering [8].

Nowadays many definitions of the fractional derivatives are introduced but most of them are in the integral form which is more complicated. The two most Known of them are the following:
Definition 1.1: [5]

1) Riemann - Liouville Definition: If $\mathrm{n}$ is a positive integer and $\alpha \in[n-1, n]$, the $\alpha^{\text {th }}$ derivative of $\mathrm{f}$ is given by

$D_{a}^{\alpha}(f)(t)=\frac{1}{\Gamma(n-\alpha)} \frac{d^{n}}{d t^{n}} \int_{a}^{t} \frac{f(x)}{(t-x)^{\alpha-n+1}} d x$

2) Caputo Definition. For $\alpha \in[n-1, n]$, the $\alpha^{\text {th }}$ derivative of $f$ is:

$D_{a}^{\alpha}(f)(t)=\frac{1}{\Gamma(n-\alpha)} \int_{a}^{t} \frac{f^{(n)}(x)}{(t-x)^{\alpha-n+1}} d x$

Now the two definitions satisfy the linearity properties of the classical derivative, but the properties of products quotient and chain rule are not satisfied where:

$D^{\alpha}(f . g) \neq$ f. $D^{\alpha} g+g . D^{\alpha} f A n d D^{\alpha}\left(\frac{f}{g}\right) \neq \frac{g^{\alpha} f-f D^{\alpha} g}{g^{2}}$ or $D^{\alpha}(f(g(x)) \neq$ $D^{\alpha} f(g(x)) \cdot D^{\alpha} g(x)$

\section{Conformable fractional derivative}

Definition2.1: [2] given a functionf: $[0, \infty) \rightarrow \mathbb{R}$. Then the Conformable fractional derivative of order $\alpha \in(0,1)$ for $f$ is defined as:

$T_{\alpha}(f)(x)=\lim _{\epsilon \rightarrow 0} \frac{f\left(x+\epsilon x^{1-\alpha}\right)-f(x)}{\epsilon}$, for all $x \in(0, \infty)$

If $f(x)$ is $\alpha$ - differentiable in some $(0, a), a>0$, and $\lim _{x \rightarrow 0+} T_{\alpha}(x)$ exist, then we define $T_{\alpha}(f)(0)=\lim _{x \rightarrow 0+} T_{\alpha}(x)$

Theorem2.1: [2] Given $f(x)$ and $g(x)$ are $\alpha$-differentiable where $\alpha \in(0,1]$ then for all $x>0$,

1) $\mathrm{T}_{\alpha}(\mathrm{af}+\mathrm{bg})(\mathrm{x})=\mathrm{a}\left(\mathrm{T}_{\alpha} \mathrm{f}\right)(\mathrm{x})+\mathrm{b}\left(\mathrm{T}_{\alpha} \mathrm{g}\right)(\mathrm{x})$ For all $\mathrm{a}, \mathrm{b} \in \mathbb{R}$.

2) $\mathrm{T}_{\alpha}\left(\mathrm{x}^{\mathrm{p}}\right)=\mathrm{px} \mathrm{x}^{\mathrm{p}-\alpha}$, for all $\mathrm{p} \in \mathbb{R}$

3) $\mathrm{T}_{\alpha}(\lambda)=0$ for all constant $\lambda \in \mathbb{R}$ 
4) $\mathrm{T}_{\alpha}(\mathrm{f} . \mathrm{g})(\mathrm{x})=\mathrm{f}(\mathrm{x})\left(\mathrm{T}_{\alpha} \mathrm{g}\right)(\mathrm{x})+\mathrm{g}(\mathrm{x}) \mathrm{T}_{\alpha}(\mathrm{f})(\mathrm{x})$

5) $\mathrm{T}_{\alpha}\left(\frac{\mathrm{f}}{\mathrm{g}}\right)(\mathrm{x})=\frac{\mathrm{g}(\mathrm{x})\left(\mathrm{T}_{\alpha} \mathrm{f}\right)(\mathrm{x})-\mathrm{f}(\mathrm{x})\left(\mathrm{T}_{\alpha} \mathrm{g}\right)(\mathrm{x})}{\mathrm{g}^{2}}$

6) Important formula will convert the CFD into classical derivative $T_{\alpha}(f)(x)=x^{1-\alpha} \frac{d f}{d x}$.

All proves for the above parts of the theorem come directly from definition 2.1 above.

7) Conformable chain rule

$\mathrm{T}_{\alpha}((\mathrm{fog})(\mathrm{x}))=\mathrm{x}^{1-\alpha} \mathrm{g}(\mathrm{x})^{1-\alpha} \mathrm{g}^{\prime}(\mathrm{x})\left(\mathrm{T}_{\alpha} \mathrm{f}\right)(\mathrm{g}(\mathrm{x}))$.

Conformable fractional derivative for some common functions:

1) $\mathrm{T}_{\alpha}\left(\mathrm{x}^{\mu}\right)=\mu \mathrm{x}^{\mu-\alpha}$,for all $\mu \in \mathbb{R}$

2) $\mathrm{T}_{\alpha}(\mathrm{a})=0$, for all $\mathrm{a} \in \mathbb{R}$

3) $\mathrm{T}_{\alpha}\left(\mathrm{e}^{\mathrm{ax}}\right)=\mathrm{ax}^{1-\alpha} \mathrm{e}^{\mathrm{ax}}$

4) $\mathrm{T}_{\alpha}(\sin (\mathrm{ax}))=\mathrm{ax}^{1-\alpha} \cos (\mathrm{ax})$

5) $\mathrm{T}_{\alpha}(\cos (\mathrm{ax}))=-\mathrm{ax}^{1-\alpha} \sin (\mathrm{ax})$

A conformable fractional derivatives of some invariant functions which are very important and arise when solving deferential equations:

1) $T_{\alpha}\left(\frac{x^{\alpha}}{\alpha}\right)=1$

2) $T_{\alpha}\left(e^{\frac{x^{\alpha}}{\alpha}}\right)=e^{\frac{x^{\alpha}}{\alpha}}$

3) $T_{\alpha}\left(\sin \left(\frac{x^{\alpha}}{\alpha}\right)\right)=\cos \left(\frac{x^{\alpha}}{\alpha}\right)$

4) $T_{\alpha}\left(\cos \left(\frac{x^{\alpha}}{\alpha}\right)\right)=-\sin \left(\frac{x^{\alpha}}{\alpha}\right)$

All above rules from 1 to 4 can be shown easily using the fact that

$T_{\alpha}(f)(x)=x^{1-\alpha} \frac{d f}{d x}$

Let us see number 3:

$\mathrm{T}_{\alpha}\left(\sin \left(\frac{\mathrm{x}^{\alpha}}{\alpha}\right)\right)=\mathrm{x}^{1-\alpha} \frac{\mathrm{d}}{\mathrm{dx}}\left(\sin \left(\frac{\mathrm{x}^{\alpha}}{\alpha}\right)\right)$

$=\mathrm{x}^{1-\alpha} \cdot \frac{\alpha \mathrm{x}^{\alpha-1}}{\alpha} \cos \left(\frac{\mathrm{x}^{\alpha}}{\alpha}\right)$

$=\cos \left(\frac{x^{\alpha}}{\alpha}\right)$ Done.

\section{Conformable fractional integral}

Definition 3.1: $[2] \quad$ let, $f(x):[a, \infty) \rightarrow \mathbb{R}$, then $I_{\alpha}^{a}(f)(x)=$ $\int_{a}^{x} \frac{f(s)}{s^{1-\alpha}} d s, \alpha \in(0,1)$ Where the integral is the usual Riemann improper integral.

Theorem3.1: $T_{\alpha} I_{\alpha}^{a}(f)(x)=f(x)$ for all $x \in(a, \infty)$ where $f$ is con tinuous in the domain ofI $I_{\alpha}$.

Again the proof is easy by using the above fact or see [2]

\section{Conformable $\mathbf{n}^{\text {th }}$ derivative}

Denote the $\alpha-$ nth derivative off(x): $(0, \infty) \rightarrow \mathbb{R}$, as $\mathrm{T}_{\alpha}^{(\mathrm{n})}(\mathrm{f})(\mathrm{x})=$ $\mathrm{T}_{\alpha} \mathrm{T}_{\alpha} \mathrm{T}_{\alpha} \ldots \ldots \ldots \mathrm{T}_{\alpha} \mathrm{f}(\mathrm{x})$

Now consider that $f(x):(0, \infty) \rightarrow \mathbb{R}$ be twice differentiable on $(0, \infty)$ and $0<\alpha<1, \beta \leq 1$ such that,

$1<\alpha+\beta \leq 2$ Then [3]:

Proposition4.1: $T_{\alpha} T_{\alpha}(f)(x)=(1-\alpha) x^{1-2 \alpha} f^{\prime}(x)+x^{2-2 \alpha} f^{\prime \prime}(x)$

Proof: $\mathrm{T}_{\alpha} \mathrm{T}_{\alpha}(\mathrm{f})(\mathrm{x})=\mathrm{T}_{\alpha}\left(\mathrm{T}_{\alpha}(\mathrm{f})(\mathrm{x})\right)=\mathrm{T}_{\alpha}\left(\mathrm{x}^{1-\alpha} \mathrm{f}^{\prime}(\mathrm{x})\right)$

$=T_{\alpha}\left(x^{1-\alpha}\right) f^{\prime}(x)+x^{1-\alpha} T_{\alpha}\left(f^{\prime}\right)(x)$

$=(1-\alpha) \mathrm{x}^{1-2 \alpha} \mathrm{f}^{\prime}(\mathrm{x})+\mathrm{x}^{2-2 \alpha} \mathrm{f}^{\prime \prime}(\mathrm{x})$
Proposition4.2: $\mathrm{T}_{\alpha}^{(3)}(\mathrm{f})(\mathrm{x})=(1-\alpha)(1-2 \alpha) \mathrm{x}^{1-3 \alpha} \mathrm{f}^{\prime}(\mathrm{x})+(3-$ $3 \alpha) \mathrm{x}^{2-3 \alpha} \mathrm{f}^{\prime \prime}(\mathrm{x})+\mathrm{x}^{3-3 \alpha} \mathrm{f}^{\prime \prime \prime}(\mathrm{x})$

Proof: Same as above.

Proposition4.3:

$T_{\alpha} T_{\beta}(f)(x)=(1-\beta) x^{1-(\alpha+\beta)} f^{\prime}(x)+x^{2-(\alpha+\beta)} f^{\prime \prime}(x)$,

Proof: $T_{\alpha}\left(T_{\beta} f\right)(x)=T_{\alpha}\left(x^{1-\beta} f^{\prime}(x)\right)$

$=T_{\alpha}\left(x^{1-\beta}\right) f^{\prime}(x)+x^{1-\beta} T_{\alpha}\left(f^{\prime}(x)\right)$

$=(1-\beta) x^{1-\beta-\alpha} f^{\prime}(x)+x^{1-\beta} x^{1-\alpha} f^{\prime \prime}(x)$

$=(1-\beta) x^{1-(\alpha+\beta)} f^{\prime}(x)+x^{2-(\alpha+\beta)} f^{\prime \prime}(x)$ Done.

if $\alpha=\beta=1$, then $T_{1} T_{1}=f^{\prime \prime}(x)$.

Note: It is easy from proposition 4.3 to show that: $\mathrm{T}_{\alpha} \mathrm{T}_{\beta}(\mathrm{f})(\mathrm{x}) \neq$ $\mathrm{T}_{\beta} \mathrm{T}_{\alpha}(\mathrm{f})(\mathrm{x})$, where $\alpha \neq \beta$.

\section{Series Solution for CFD}

In this section we will discuss the series solution of CFD for FDE. Note that the power series in powers of $(x-a)$ is an infinite series of the form [4], [5]:

$\sum_{n=0}^{\infty} a_{n}(x-a)^{n \alpha}=a_{0}+a_{1}(x-a)^{\alpha}+a_{2}(x-a)^{2 \alpha}+\cdots+$ $\mathrm{a}_{\mathrm{n}}(\mathrm{x}-\mathrm{a})^{\mathrm{n} \alpha}+\cdots \ldots$. $^{*}$

If $\mathrm{a}=0$ the series become:

$\sum_{n=0}^{\infty} a_{n} x^{n \alpha}=a_{0}+a_{1} x^{\alpha}+a_{2} x^{2 \alpha}+\cdots+a_{n} x^{n \alpha}+\cdots \ldots \ldots . * *$

If the power series in $(* *)$ converges on the interval $I=(0, \infty)$, this means that:

$\sum_{n=0}^{\infty} a_{n} x^{n \alpha}=\lim _{N \rightarrow \infty} \sum_{n=0}^{N} a_{n} x^{n \alpha}$, exist for each $x \in I$ in this case the below sum

$\mathrm{f}(\mathrm{x})=\sum_{\mathrm{n}=0}^{\infty} \mathrm{a}_{\mathrm{n}} \mathrm{x}^{\mathrm{n} \alpha}$ Is defined on I which is a power series representation of $\mathrm{f}(\mathrm{x})$ onI.

- To study the convergence of the fractional power series we test that by ratio test as follows:

$\lim _{n \rightarrow \infty}\left|\frac{a_{n} x^{n \alpha}}{a_{n+1} x^{n \alpha+\alpha}}\right|=\lim _{n \rightarrow \infty}\left|\frac{a_{n}}{a_{n+1} x^{\alpha}}\right|$

Now if we place $r=\lim _{n \rightarrow \infty}\left|\frac{a_{n}}{a_{n+1}}\right|$ then:

1) If $\mathrm{r}=0$, the series will diverges for all $\mathrm{x} \neq 0$

2) If $0<\mathrm{r}<\infty$, then $\sum_{\mathrm{n}=0}^{\infty} \mathrm{a}_{\mathrm{n}} \mathrm{x}^{\mathrm{n} \alpha}$ is converges if $|\mathrm{x}|<\mathrm{r}^{\frac{1}{\alpha}}$ and diverges if $|x|>r^{\frac{1}{\alpha}}$.

3) If $r=\infty$ the series converges for all $x$.

Remark: Term wise Conformable differentiation of power series

If $f(x)=\sum_{n=0}^{\infty} a_{n} x^{n \alpha}=a_{0}+a_{1} x^{\alpha}+a_{2} x^{2 \alpha} \ldots$, Then:

1) $\left(T_{\alpha} f\right)(x)=\sum_{n=1}^{\infty} n \alpha a_{n} x^{n \alpha-\alpha}=\sum_{n=1}^{\infty} n \alpha a_{n} x^{(n-1) \alpha}$.

2) $\left(\mathrm{T}_{\alpha} \mathrm{T}_{\alpha} \mathrm{f}\right)(\mathrm{x})=\sum_{\mathrm{n}=2}^{\infty} \mathrm{n} \alpha(\mathrm{n} \alpha-\alpha) \mathrm{x}^{\mathrm{n} \alpha-2 \alpha}=$ $\sum_{n=2}^{\infty} \alpha^{2} n(n-1) a_{n} x^{(n-2) \alpha}$. 


\section{Riccati conformable fractional differential equation with variable coefficients}

Consider the CFDE:

$y^{(2 \alpha)}+x^{\alpha} y^{(\alpha)}+x^{2 \alpha} y=0$

Where $\alpha \in(0,1)$ and $x>0$, consider the initial conditions

$$
y(0)=0, y^{(\alpha)}(0)=1
$$

We will denote:

$\left(T_{\alpha} y\right)(x)=y^{(\alpha)}(x), T_{\alpha} T_{\alpha} y(x)=y^{(2 \alpha)}(x)$.

First of all we need to show that this is a one case of Ricati differential equation with variable coefficients: [9]

To show that we will use the following change of variables:

$z(x)=-\frac{y^{(\alpha)}(x)}{y(x)}$

Then: $y^{(\alpha)}(x)=-z(x) y(x)$

Now with some calculations and using the Theorem1 part 5 we get:

$y^{(2 \alpha)}=y z^{(\alpha)}-z^{2} y$

Substitute (2), (3) and (4) in (1) we get:

$z^{(\alpha)}(x)=x^{2 \alpha}-z^{2}(x)-x^{\alpha} z(x)$

Which is a nonlinear Ricati conformable differential equation with variable coefficients?

Now we need to use a series solution to find a general solution of (1).

$$
\begin{aligned}
& \text { let } y=\sum_{n=0}^{\infty} a_{n} x^{n \alpha} \text { So, } \\
& x^{2 \alpha} y=\sum_{n=0}^{\infty} a_{n} x^{\alpha(n+2)} \\
& \text {, then } y^{(\alpha)}=\sum_{n=1}^{\infty} n \alpha a_{n} x^{\alpha(n-1)} \text { So, } \\
& x^{\alpha} y^{(\alpha)}=\sum_{n=1}^{\infty} n \alpha a_{n} x^{n \alpha} \\
& y^{(2 \alpha)}=\sum_{n=2}^{\infty} \alpha^{2} n(n-1) a_{n} x^{\alpha(n-2)}
\end{aligned}
$$

Now in (6) replace $(n)$ by $(n+2)$ we get: $x^{2 \alpha} y=\sum_{n=2}^{\infty} a_{n-2} x^{\alpha n}$ In (7) no need to replace parameters: $x^{\alpha} y^{(\alpha)}=\sum_{n=1}^{\infty} n \alpha a_{n} x^{n \alpha}$ In (8) replace $(n)$ by $(n-2)$ we get:

$y^{(2 \alpha)}=\sum_{n=0}^{\infty} \alpha^{2}(n+2)(n+1) a_{n+2} x^{\alpha n}$
Now substitute the last three results in the equation (1) we get that: $\sum_{n=0}^{\infty} \alpha^{2}(n+2)(n+1) a_{n+2} x^{\alpha n}+\sum_{n=1}^{\infty} n \alpha a_{n} x^{n \alpha}+$

$\sum_{n=2}^{\infty} a_{n-2} x^{\alpha n}=0$

In the last equation we will unify all summations to start from

$(n=2)$ So:

$2 \alpha^{2} a_{2}+6 \alpha^{2} a_{3} x^{\alpha}+\alpha a_{1} x^{\alpha}+\sum_{n=2}^{\infty} \alpha^{2}(n+2)(n+1) a_{n+2} x^{\alpha n}+$ $\sum_{n=2}^{\infty} n \alpha a_{n} x^{n \alpha}+\sum_{n=2}^{\infty} a_{n-2} x^{\alpha n}=0$

From this we find that:

1) $2 \alpha^{2} a_{2}=0 \rightarrow a_{2}=0$

2) $6 \alpha^{2} a_{3}+\alpha a_{1}=0$, then: $a_{3}=\frac{-1}{6 \alpha} a_{1}$

3) $\alpha^{2}(n+2)(n+1) a_{n+2}+n \alpha a_{n}+a_{n-2}=0$, then:

$$
a_{n+2}=\frac{-n \alpha a_{n}-a_{n-2}}{\alpha^{2}(n+2)(n+1)}
$$

Where (11) is a three-term recurrence relation where $n \geq 2$.From (11) we get:

$a_{4}=\frac{-1}{12 \alpha^{2}} a_{0}, a_{6}=\frac{-1}{\alpha^{3} 45} a_{0}$,

$a_{8}=\frac{3}{1120 \alpha^{4}} a_{0}, a_{10}=\frac{-17}{113400 \alpha^{5}} a_{0}, \ldots \ldots$

$a_{5}=\frac{-1}{40 \alpha^{2}} a_{1}, a_{7}=\frac{1}{144 \alpha^{3}} a_{1}$,

$a_{9}=\frac{-17}{51840 \alpha^{4}} a_{1}, a_{11}=\frac{-53}{633600 \alpha^{5}} a_{1}, \ldots \ldots$

Now in case that we have an odd and even terms let us apply the initial conditions in the equation solution

$y=\sum_{n=0}^{\infty} a_{n} x^{n \alpha}$

$y(0)=0 \rightarrow a_{0}=0, y^{\alpha}(0)=1$, then: $a_{1}=\frac{1}{\alpha}$

According to these results all even terms will be canceled and the odd terms will remain, so the solution will become:

$\mathrm{y}(\mathrm{x})=\frac{1}{\alpha} \mathrm{x}^{\alpha}-\frac{1}{6 \alpha^{2}} \mathrm{x}^{3 \alpha}-\frac{1}{40 \alpha^{3}} \mathrm{x}^{5 \alpha}+\frac{1}{144 \alpha^{4}} \mathrm{x}^{7 \alpha}-\frac{17}{51840 \alpha^{5}} \mathrm{x}^{9 \alpha}-$ $\frac{53}{633600 \alpha^{6}} \mathrm{x}^{11 \alpha}+\ldots$

If we consider only the first 6 terms of this series, we will get the following graph for several values of $\alpha$. 


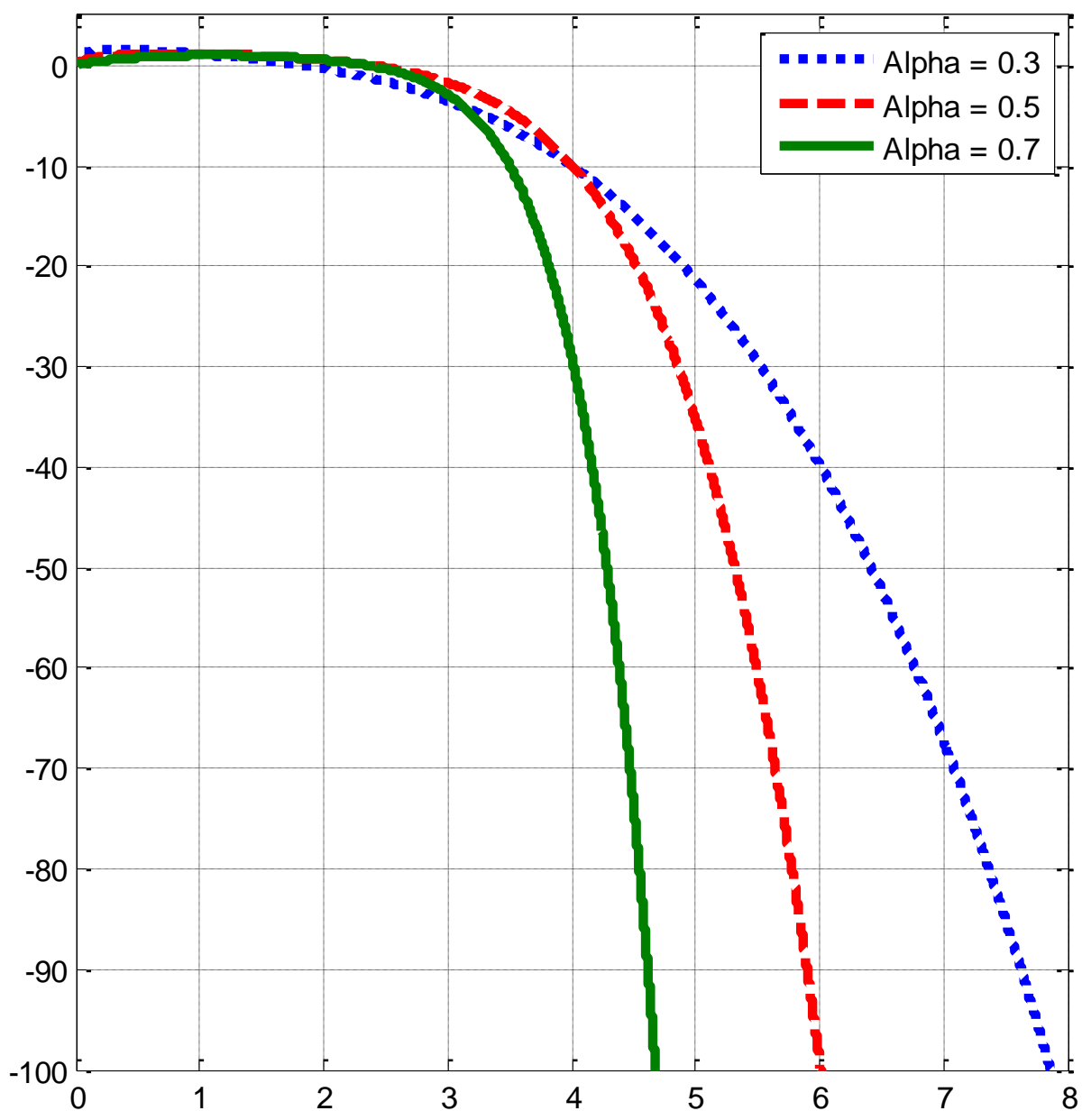

Now consider other initial conditions $\mathrm{y}(0)=1, \mathrm{y}^{\alpha}(0)=0$ here the odd terms will be all cancelled and since $\mathrm{a}_{1}=0$ and $\mathrm{a}_{0}=1$ the solution become: $y(x)=1-\frac{1}{12 \alpha^{2}} x^{4 \alpha}-\frac{1}{45 \alpha^{3}} x^{6 \alpha}+\frac{3}{1120 \alpha^{4}} x^{8 \alpha}-$ $\frac{17}{113400 \alpha^{5}} x^{10 \alpha}+\cdots$

\section{Conclusion}

In this paper, we state some important properties for the conformable fractional derivative of multi order, which can lead to converting the fractional order differential equations into ordinary differential equations. And investigate series solutions of Riccati differential equation with variable coefficients, which is not easy to find an exact solution.

\section{Acknowledgments}

I would like to thank prof. Roshdi Khalil for introducing me to the field of Fractional calculus and all of his referees for his suggestions, which improve the article to its present form.

\section{References}

[1] K.Assaleh; W. M.Ahmed, Modeling of speech signals using fractional calculus 9th international symposium on Signal Processing and its Applications, 2007.ISSPA 2007.12-15 Feb. 2007 Page: 1-4.

[2] R. Khalil, M. A. Horani, A. Youssef and M. Sababheh, A new definition of fractional derivative, J. Compute. Appl. Math. 264, 65-70 (2104).
[3] Abdeljawad, Thabet, on conformable fractional calculus, J. Computational. Applied. Math. 279(2015) 57-66. https://doi.org/10.1016/j.cam.2014.10.016.

[4] A. El-Ajou, O. Abu Arqub, Z. Al Zhour and S. Momani, New Results on Fractional Power Series: Theories and Applications, Entropy 2013, 15, 5305-5323; https://doi.org/10.3390/e15125305.

[5] K.S. Miller, An introduction to fractional calculus and Fractional differential Equations, J. Wiley and Sons, New York, 1993.

[6] Bagley RL, Torvik PJ. A theoretical basis for the application of fractional calculus to viscoelasticity. J Rheol 1983; 27(3):201-10. https://doi.org/10.1122/1.549724.

[7] Hartley TT, Lorenzo CF, Qammar HK. Chaos in a fractional order Chua system. IEEE Trans. Circ. Syst. I1995; 42(8):485-90.

[8] Magin RL. Fractional calculus in bioengineering. Crit. Rev. Biomed Eng. 2004; 32(1):1-104. https://doi.org/10.1615/CritRevBiomedEng.v32.10.

[9] K. Busawon, P. Johnson, Solution of a class of Riccati Equations, WSEAS International Conference on Applied Mathematics, Tenerife, Spain, Dec 16-18,2005(pp334-338). 